\title{
Validation Study of a Contactless Monitoring Device for Vital Signs During Sleep and Sleep Architecture in Adults With Sleep-Disordered Breathing
}

\author{
Young Jeong Lee, $\mathrm{MD}^{1}$, Hyun Tag Kang, MD ${ }^{1}$, Ji Ho Choi, MD, $\mathrm{PhD}^{1}$, Ji Eun Moon, $\mathrm{PhD}^{2}$, \\ Young Jun Lee, $\mathrm{PhD}^{3}$, Tae Kyung Ha ${ }^{3}$, Ho Dong Lee ${ }^{3}$ \\ ${ }^{1}$ Department of Otorhinolaryngology-Head and Neck Surgery, Soonchunhyang University Bucheon Hospital, \\ Soonchunhyang University College of Medicine, Bucheon, Korea \\ ${ }^{2}$ Department of Biostatistics, Clinical Trial Center, Soonchunhyang University Bucheon Hospital, Bucheon, Korea \\ ${ }^{3}$ Honeynaps Research and Development Center, Honeynaps Co. Ltd., Seoul, Korea
}

\author{
Received: November 19, 2021 \\ Revised: December 15, 2021 \\ Accepted: December 23, 2021 \\ Correspondence \\ Ji Ho Choi, MD, PhD \\ Department of Otorhinolaryngology-Head \\ and Neck Surgery, \\ Soonchunhyang University Bucheon Hospital, \\ Soonchunhyang University College of Medicine, \\ 170 Jomaru-ro, Bucheon 14584, Korea \\ Tel +82-32-621-5015 \\ Fax +82-32-621-5016 \\ E-mail handsomemd@hanmail.net
}

\section{ORCID}

Young Jeong Lee

http://orcid.org/0000-0003-1273-1946

Hyun Tag Kang

http://orcid.org/0000-0003-4549-8079

Ji Ho Choi

http://orcid.org/0000-0002-5194-930X

Ji Eun Moon

http://orcid.org/0000-0002-7293-7463

Young Jun Lee

http://orcid.org/0000-0002-6135-7834

Tae Kyung Ha

http://orcid.org/0000-0002-1303-8724

Ho Dong Lee

http://orcid.org/0000-0002-2611-9478

(c) This is an Open Access article distributed under the terms of the Creative Commons Attribution Non-Commercial License (https://creativecommons.org/licenses/by-nc/4.0) which permits unrestricted non-commercial use, distribution, and reproduction in any medium, provided the original work is properly cited.
Background and Objective Few clinical studies have investigated the accuracy of non-contact monitoring devices for vital signs during sleep and sleep architecture in adults with sleep-disordered breathing (SDB). The purpose of this study was to assess the accuracy of a contactless monitoring device for 1) heart rate, respiratory rate, and body temperature during sleep and 2) sleep architecture in adults with SDB.

Methods Thirty-five consecutive adults, who visited a tertiary university hospital due to suspected SDB, underwent a complete physical examination and standard (level 1) polysomnography plus body temperature measurement with a contactless monitoring device (HoneyCube System).

Results A total of 30 subjects (mean age $=46.43 \pm 12.9$ years; male: female $=22$ : 8 ) were finally included, and five subjects were excluded due to inadequate data in this study. The intraclass correlation coefficient values of heart rate, respiratory rate, and body temperature measured using the contactless monitoring device were 0.91 (95\% confidence interval [CI]: 0.892, 0.928), 0.937 (95\% CI: $0.919,0.954)$, and 0.918 (95\% CI: 0.895, 0.941), respectively. The mean kappa value for sleep architecture was 0.562 (95\% CI: 0.529, 0.596).

Conclusions The contactless monitoring device showed good (almost perfect) agreement in terms of heart rate, respiratory rate, and body temperature and moderate agreement in sleep architecture with contact measurements. These results suggest that the HoneyCube System is a good candidate device for sleep monitoring at home and in multiple accommodations.

Sleep Med Res 2021;12(2):118-124

Keywords Sleep-disordered breathing; Heart rate; Respiratory rate; Body temperature; Sleep stages.

\section{INTRODUCTION}

Vital signs reflect the most critical medical signs that imply the condition of the body's vital functions or life-sustaining activities [1]. Clinically, vital signs can be used as indicators to judge or evaluate an individual's health status. For example, vital signs usually include heart rate, respiratory rate, and body temperature.

Sleep-disordered breathing (SDB) is defined as a group of disorders characterized by repeated episodes of upper airway narrowing or obstruction during sleep [2]. It includes conditions of abnormal respiration or respiratory disturbance during sleep, ranging from simple snoring to obstructive sleep apnea (OSA). SDB is relatively common, and its prevalence tends to increase with age or weight gain [3]. There are various pathophysiological mechanisms related to symp- 
toms and complications of SDB, including intermittent hypoxia, hypercapnia, intrathoracic pressure swings, hyperactivity of the sympathetic nervous system, and sleep fragmentation [4]. SDB may induce numerous symptoms such as excessive daytime sleepiness, loud or habitual snoring, pauses in respiration during sleep, morning headaches, poor concentration, and decreased quality of life [5]. If SDB is neglected or persists, serious complications, including hypertension, elevated insulin resistance, coronary artery disease, arrhythmia, and stroke, can occur [6]. Thus, it is very necessary and important to monitor vital signs in patients with SDB during sleep.

Conventionally, vital signs are measured through direct contact. However, with recent developments in medical science and technology, diverse non-contact or contactless methods have been developed to measure or monitor vital signs. Among these methods, ballistocardiography is a useful technique for detecting vital signs. Ballistocardiography is a technology that graphically represents the repeated movements of the human body caused by the sudden ejection of blood into large blood vessels with each heartbeat [7]. The mechanisms of ballistocardiography in the detection of body motion caused by cardiac activity are as follows [8]: 1) the heart beats and induces blood entry into the aortic vessels. 2) The blood that has pushed into the aortic vessels reacts to the blood flow, resulting in dilation and contraction. 3) This leads to weak, cyclic, involuntary body movements that can be measured by ballistocardiography. In addition, ballistocardiography can detect body motion caused by respiratory activity based on similar mechanisms. Moreover, sleep architecture can be analyzed by making an algorithm using cardiac and respiratory activity measured by ballistocardiography [9].

Many studies have been conducted to measure vital signs such as heart rate and respiratory rate using ballistocardiography in a non-contact manner $[8,10,11]$. However, studies that analyzed sleep architecture using ballistocardiography or studies that measured vital signs using a non-contact method for SDB patients are insufficient. Therefore, the aim of this study was to evaluate the accuracy of a contactless monitoring device for 1) heart rate, respiratory rate, and body temperature during sleep and 2) sleep architecture in adults with SDB.

\section{METHODS}

\section{Subjects}

This study was conducted prospectively for 35 consecutive adults who visited a tertiary university hospital between October and December 2019 due to suspected SDB. All subjects underwent a complete physical examination and standard (level 1) polysomnography with a contactless monitoring device. The body mass index (BMI) of each participant was analyzed using their weight in kilograms divided by their height in meters squared $\left(\mathrm{kg} / \mathrm{m}^{2}\right)$. This clinical investigation protocol was reviewed and approved by the Institutional Review Board (IRB) of Soonchunhyang University Bucheon Hospital (SCHBC 2018-08-021). The written informed consent was obtained from all participants.

\section{Polysomnographic Examination and Contact Body Temperature Measurement}

An attended full-night polysomnography was performed in all participants based on a computerized polysomnographic system (Embla N7000; Natus Medical Inc., San Carlos, CA, USA). The polysomnographic examination parameters included a two-channel electrooculogram, six-channel electroencephalogram, submental and leg electromyograms, electrocardiogram (ECG), airflow (thermistor and pressure transducer), oxygen saturation, respiratory effort (chest and abdominal movement), snoring, and body position. A sleep technician monitored all subjects' bio-signals overnight and manually scored all raw data according to the American Academy of Sleep Medicine scoring manual [12]. All scored raw data were reviewed by a certified sleep specialist. Sleep efficiency was defined as the ratio of total sleep time (the total amount of time spent in sleep stages) to the total recording time (the total amount of time from 'lights out' to lights on'). The arousal index was defined as the number of arousals per hour of total sleep time. The percentage of total sleep time in each sleep stage (N1, N2, N3, and R), known as sleep architecture, was defined as the ratio of time in each stage to the total sleep time. The apnea-hypopnea index was defined as the number of apnea events (absence of airflow $\geq 10$ seconds) and hypopnea (decrease of airflow $\geq 30 \%$ related to oxygen desaturation $\geq 3 \%$ or arousal) per hour of total sleep time. The oxygen desaturation index was defined as the number of events of oxygen desaturation $\geq 3 \%$ per hour of total sleep time. Snoring was defined as the ratio of snoring time to total sleep time. Body temperature measurements were performed in all subjects using a commercial patient monitoring device (BT-740; Bistos Co. Ltd., Seongnam, Korea).

\section{Heart Rate, Respiratory Rate, Body Temperature, and Sleep Architecture Measurements Using Contactless Monitoring Devices}

Bio-signals including heart rate, respiratory rate, body temperature, and sleep architecture were measured in all participants using a contactless monitoring device (HoneyCube System; Honeynaps Co. Ltd., Seoul, Korea) (Fig. 1). The HoneyCube system consists of two parts: a head side unit (body temperature and video module) and a bedside unit (heart and respiratory rate module). The head side unit was installed $50 \mathrm{~cm}$ above the center of the head of the patient's bed, and the bedside unit was installed $30 \mathrm{~cm}$ from the top left of the bed. Heart rate, respiration rate, and body temperature were measured primarily based on non-contact methods (heart and respiratory rate were measured by ballistocardiography) during sleep, and sleep architecture was secondarily calculated by an algorithm combining heart rate and 

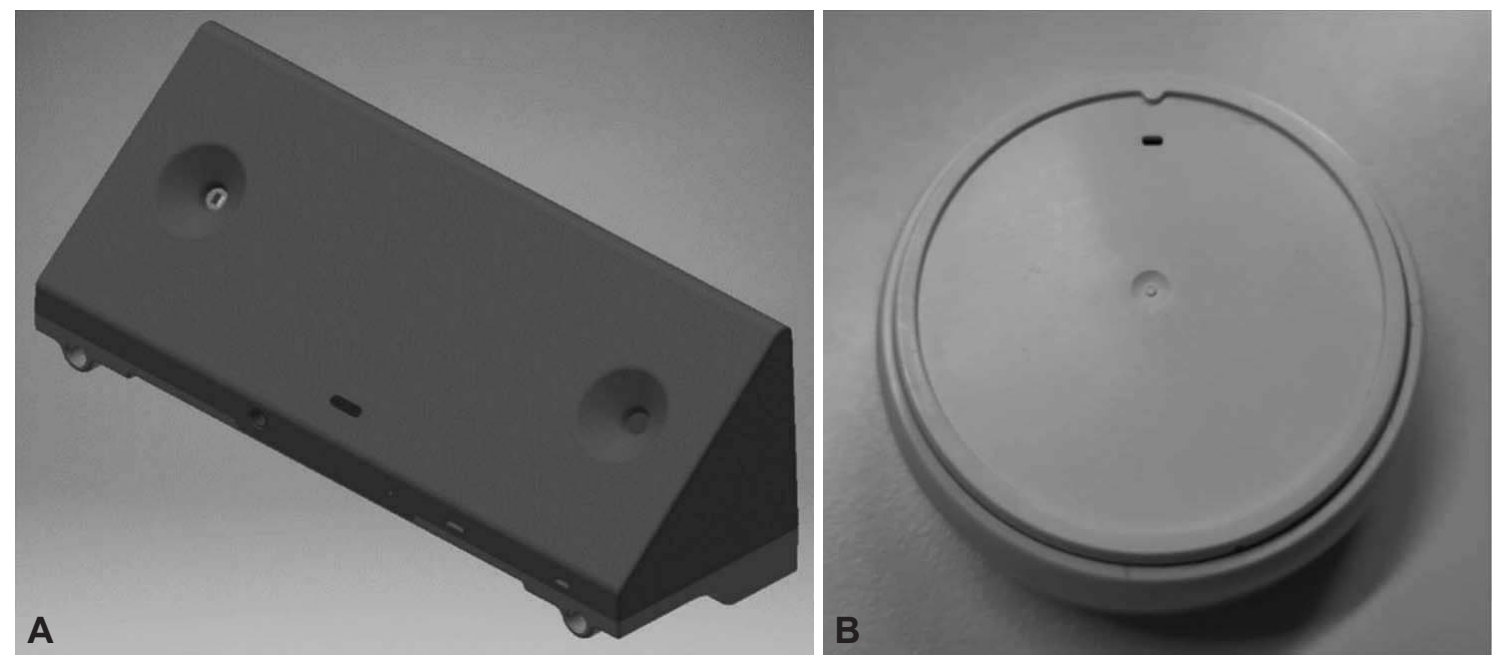

Fig. 1. The contactless monitoring device (HoneyCube System) consists of two parts including the (A) body temperature \& video module and $(B)$ heart \& respiratory rate module.

respiration rate. In this study, the sampling rate of heart rate and respiratory rate was $1 \mathrm{~Hz}$, that of body temperature was $1 / 60 \mathrm{~Hz}$, and sleep stages were scored with $30 \mathrm{sec}$ representing single epochs.

\section{Algorithm for Automatic Sleep Stage Scoring}

A deep learning algorithm was used to analyze sleep stages. Heart rate, heart rate variability, respiratory rate, and respiratory rate variabilities extracted from ballistocardiography were used as inputs. To build a base deep learning model, Sleep Heart Health Study data were used, and then a transfer learning method was used to build a final deep learning model for ballistocardiography data. The model for sleep stage scoring consists of five layers: first, a perceptron layer, three consecutive layers of long shortterm memory (LSTM) cells, and finally, two more perceptron layers. The first perceptron layer consists of perceptrons, each generating a linear combination of all features. Each LSTM layer consists of $\mathrm{N}$ cells: $\mathrm{N} / 2$ moves in the forward direction, passing their internal values to future epochs, while the other cells pass values in the opposite direction. Finally, the $\mathrm{N}$ values coming out of the last LSTM layer are processed by a perceptron layer with $\mathrm{N} / 2$ neurons and a final subsequent layer with four neurons corresponding to the four class probabilities. All activation functions used were sigmoid, with the exception of the last layer, where a softmax activation function was used.

\section{Statistical Analysis}

In this study, Cohen's kappa $(\kappa)$ statistic was used to evaluate the agreement between the category measurements by our proposed model (sleep architecture). Kappa statistics were interpreted using the following criteria (Landis and Koch [13]): values of $0.00-0.20,0.21-0.40,0.41-0.60,0.61-0.80$, and $0.81-1.00$ indicated slight, fair, moderate, substantial, and almost perfect agreement, respectively. The intraclass correlation coefficient (ICC) as defined by McGraw and Wong [14] was used to evaluate the reliability of the scales with continuous variables using a twoway mixed model (heart rate, respiratory rate, and body temperature). The interpretation of ICC was the same as that of kappa. The box-and-whisker plot and jitter plot show the distribution of the Kappa and ICC values for 30 observations. All statistical analyses were performed using R version 3.6.3 (The R Foundation for Statistical Computing, Vienna, Austria) and SPSS Statistics version 26.0 (IBM Corp., Armonk, NY, USA).

\section{RESULTS}

A total of 30 subjects (mean age $=46.43 \pm 12.9$ years; 22 men and 8 women; BMI $\left.\left[\mathrm{kg} / \mathrm{m}^{2}\right]=26.26 \pm 3.37\right)$ with SDB were finally included in this study. Five subjects were excluded from this analysis due to inadequate data. The polysomnographic data from three subjects was excluded because breathing movement and respiratory signals could not be detected due to patient movements, and two patients were excluded because ballistocardiography data loss occurred in some period due to wireless communication failure and data quality issues. There were 24 patients with OSA ( mild $=11$, moderate $=6$, and severe $=7$ ) and 6 patients with simple snoring. The sleep scoring data, including arousal events, of all subjects were as follows; sleep efficiency (\%), stage N1, N2, N3, R (\% of total sleep time), and arousal index (events/hour of total sleep time) values: $85.26 \pm 9.15$, $18.68 \pm 9.46,56.41 \pm 13.68,2.21 \pm 4.73,17.12 \pm 5.17$, and 27.18 \pm 13.06 , respectively. Respiratory events, including snoring, of all subjects were as follows; apnea-hypopnea index (events/hour of total sleep time), oxygen desaturation index (events/hour of total sleep time), average arterial oxygen saturation (\%), minimum arterial oxygen saturation (\%), and snoring (\%): $18.81 \pm$ $15.53,17.82 \pm 14.81,95.2 \pm 1.57,83.67 \pm 6.74$, and $44.52 \pm 22.4$, respectively. Baseline data are presented in Table 1. 


\section{Comparison of Heart Rate Between Contactless and Contact Measurements}

ICC values were used to determine the agreement in heart rate between the contactless and contact measurements. The mean ICC value for heart rate was 0.91 , and the $95 \%$ confidence interval (CI) was 0.892 to 0.928 . Box and whisker plots overlaid on the jitter plot for heart rate are shown in Fig. 2.

\section{Comparison of Respiratory Rate Between Contactless and Contact Measurements}

We performed statistical analysis based ICC values to establish the agreement in respiratory rate between contactless and contact measurements. The mean ICC value for respiratory rate was 0.937 , and the $95 \% \mathrm{CI}$ was $0.919-0.954$. Box and whisker plots overlaid on the jitter plot for the respiratory rate are presented in Fig. 3.

\section{Comparison of Body Temperature Between Contactless and Contact Measurements}

ICC values were used to identify the agreement in body temperature between the contactless and contact measurements. The mean ICC for body temperature was 0.918 , with a $95 \%$ CI of 0.895 to 0.941 . Box and whisker plots overlaid on the jitter plot for body temperature are shown in Fig. 4 .

\section{Comparison of Sleep Architecture Between Contactless and Contact Measurements}

We performed statistical analysis using Cohen's kappa $(\kappa)$ to demonstrate the agreement in sleep architecture between con-

Table 1. Baseline demographic and polysomnographic data

\begin{tabular}{lc}
\hline \multicolumn{1}{c}{ Variables } & Subjects $(\mathrm{n}=30)$ \\
\hline Age $(\mathrm{yr})$ & $46.43 \pm 12.9$ \\
Sex $(\mathrm{M} / \mathrm{F})$ & $22 / 8$ \\
Body mass index $\left(\mathrm{kg} / \mathrm{m}^{2}\right)$ & $26.26 \pm 3.37$ \\
Polysomnographic data & \\
Total sleep time (TST) (min) & $361.79 \pm 67.98$ \\
Sleep efficiency (\%) & $85.26 \pm 9.15$ \\
Arousal index (events/hour of TST) & $27.18 \pm 13.06$ \\
Stage N1 (\% of TST) & $18.68 \pm 9.46$ \\
Stage N2 (\% of TST) & $56.41 \pm 13.68$ \\
Stage N3 (\% of TST) & $2.21 \pm 4.73$ \\
Stage R (\% of TST) & $17.12 \pm 5.17$ \\
Apnea-hypopnea index (events/hour of TST) & $18.81 \pm 15.53$ \\
Oxygen desaturation index & $17.82 \pm 14.81$ \\
(events/hour of TST) & \\
Average arterial oxygen saturation (\%) & $95.2 \pm 1.57$ \\
Minimum arterial oxygen saturation (\%) & $83.67 \pm 6.74$ \\
Snoring (\% of TST) & $44.52 \pm 22.4$ \\
\hline Data are presented as mean \pm standard &
\end{tabular}

Data are presented as mean \pm standard deviation. tactless and contact measurements. The mean value of kappa for sleep architecture was 0.562 , with a $95 \%$ CI of 0.529 to 0.596 . Box and whisker plots overlaid on the jitter plot for respiratory rate are shown in Fig. 5.

\section{DISCUSSION}

This prospective validation study was conducted to investigate the agreement in vital signs and sleep stages between noncontact monitoring devices using ballistocardiography and contact measurements, including polysomnography. The results of

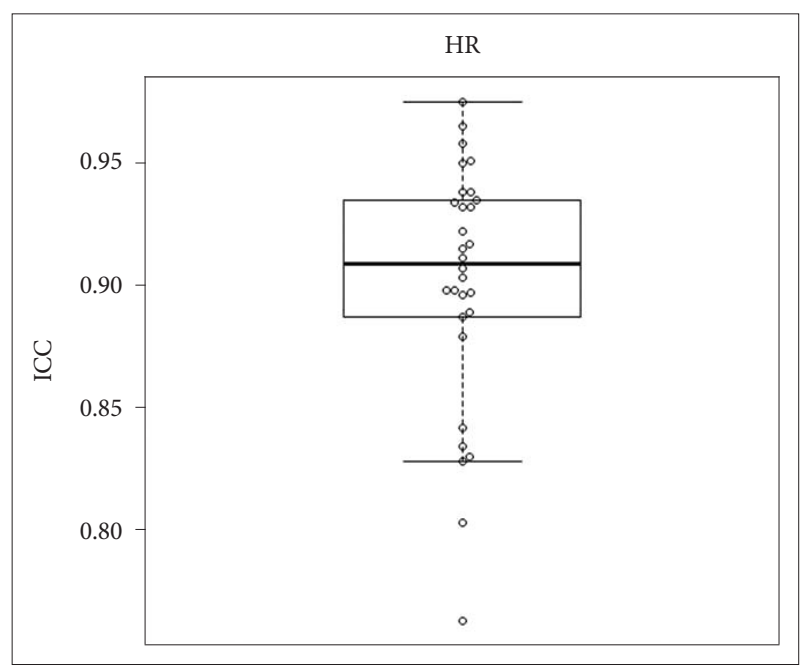

Fig. 2. Box and whisker plot overlaid upon the jitter plot for HR. The mean ICC value for HR was 0.91 (95\% confidence interval: 0.892-0.928). It is drawn from Q1 to Q3, with a horizontal line drawn in the middle to denote the median. ICC, intraclass correlation coefficient; HR, heart rate.

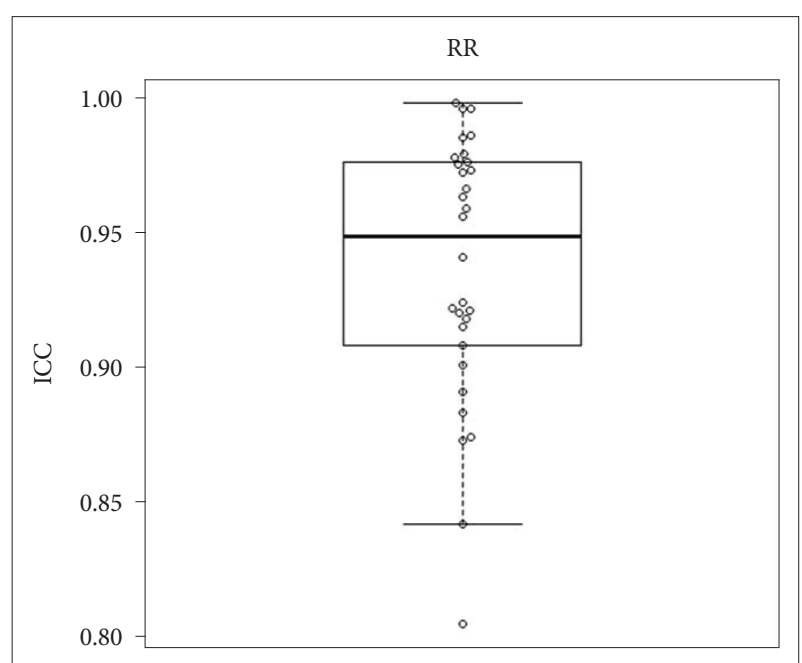

Fig. 3. Box and whisker plot overlaid upon the jitter plot for RR. The mean ICC value for RR was 0.94 (95\% confidence interval: 0.919-0.954). It was drawn from Q1 to Q3, with a horizontal line drawn in the middle to denote the median. ICC, intraclass correlation coefficient; $\mathrm{RR}$, respiratory rate. 


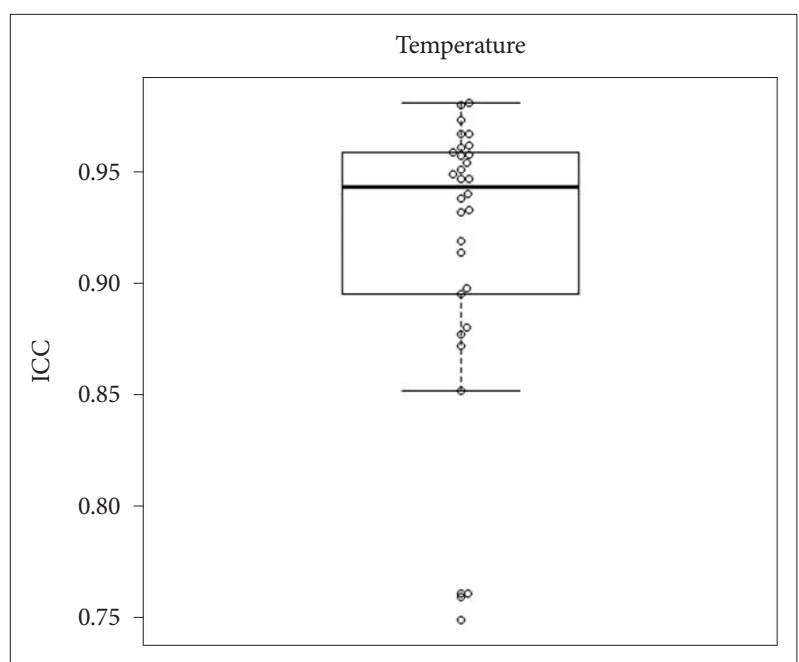

Fig. 4. Box and whisker plot overlaid upon the jitter plot for body temperature. The mean ICC value for body temperature was 0.92 (95\% confidence interval: 0.895-0.941). It was drawn from Q1 to $\mathrm{Q} 3$, with a horizontal line drawn in the middle to denote the median. ICC, intraclass correlation coefficient.

the study indicated that there was good (almost perfect) agreement in heart rate, respiratory rate, and body temperature between contactless and contact measurements. In addition, moderate agreement in sleep architecture was found between contactless and contact measurements.

Heart rate or pulse rate is the number of heartbeats per unit of time and is generally presented as beats per minute (bpm). Heart rate can vary depending on age, physical activity or fitness level, psychological status, medications, and disease. In adults, the normal range of resting heart rate is $60-100 \mathrm{bpm}$. An unnaturally slow heart rate (less than $60 \mathrm{bpm}$ ) is called bradycardia and may be associated with various conditions such as athletic lifestyles, older ages, drug use, and heart disorders. On the other hand, an abnormally fast heart rate (> $100 \mathrm{bpm})$ is called tarchycardia and may be related to diverse conditions, including normal responses (due to pain, fever, and stress), substances, medications, and medical illness (e.g., anemia, hyperthyroidism, cardiopulmonary disease, sepsis, and so on). Heart rate is the most central component in ballistocardiograms, which have become more accurate than when first introduced in several studies $[15,16]$. Xie et al. [15] compared beat-to-beat heart rate detection from ballistocardiograms for smart home applications using ECG. Their results showed an almost perfect detection of 99\% for beat-to-beat intervals. Albukhari et al. [16] also used a bed-embedded ballistocardiographic device to detect heart rate, and the overall accuracy was $83.9 \%$. When patients laid on their left sides, it showed a higher accuracy of $91 \%$.

Respiratory rate is the number of breaths (inspiration and expiration) per unit of time and is usually recorded as respirations per minute. Respiratory rate can change depending on age, exercise, emotional state, drugs, and medical conditions. The normal range of respiratory rate in adults is, on average, 16-20 breaths

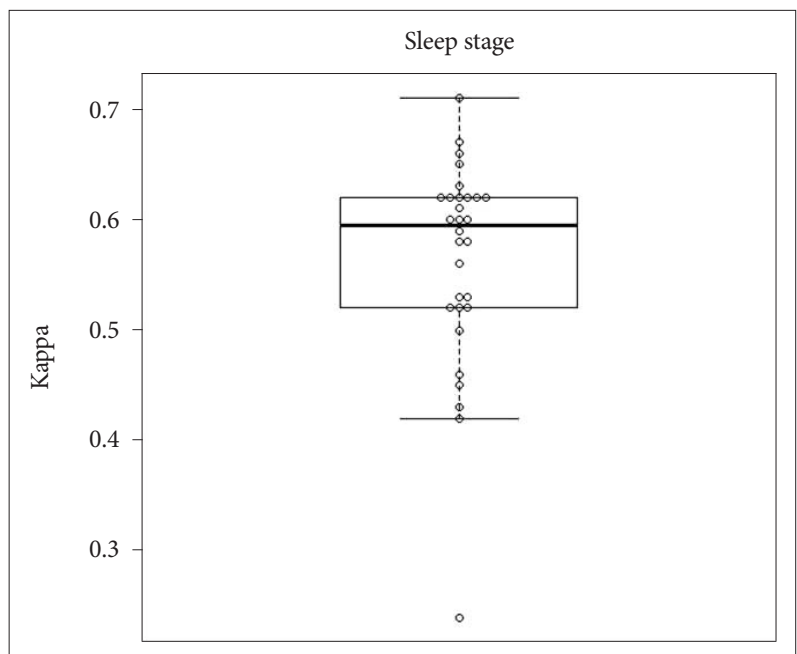

Fig. 5. Box and whisker plot overlaid upon the jitter plot for sleep stage. The mean value of Cohen's kappa for heart rate was 0.56 (95\% confidence interval: 0.529-0.596). It was drawn from Q1 to Q3, with a horizontal line drawn in the middle to denote the median. ICC, intraclass correlation coefficient.

per minute. Abnormal respiratory rates such as bradypnea and tarchypnea may be clinically caused by pain, anxiety, stress, shock, medications, and medical conditions (e.g., cerebral lesions, pulmonary heart disease, neuromuscular disorders, and so on). Sadek et al. [17] used a microbend fiber optic sensor to collect ballistocardiogram data to monitor sleep apnea and vital signs. To quantify the performance of their study, normalized mean absolute error (NMAE), normalized root mean square error (NRMSE), and mean absolute percentage error (MAPE) proportions were adopted. On average, the NMAE, NRMSE, and MAPE proportions were $5.42 \%, 6.54 \%$, and $5.41 \%$, respectively, for heart rate estimation. They showed significant agreement on the respiratory rate between the microbend fiber optic sensor and polysomographic results $(\mathrm{r}=0.41, \mathrm{p}<0.001)$. In the current study, a different type of sensor other than a microbend fiber optic sensor was used. In addition to home and medical environments, some researchers have applied ballistocardiograms in completely different environments, such as in vehicles and headsets, and have shown good results in terms of respiratory rate [18].

Sleep architecture is defined as a basic sleep structure in which the four sleep stages (stages N1, N2, N3, and R) change cyclically. Sleep stages are generally divided into rapid eye movement (REM) sleep and non-rapid eye movement (NREM) sleep, and NREM sleep is classified into stages N1, N2, and N3, indicating a continuity of relative depth. Sleep architecture can be affected by age, inappropriate sleep duration, drugs, and medical conditions. Abnormal sleep architecture may be clinically associated with short or long sleep, medications, and diverse sleep disorders (e.g., insomnia, OSA, periodic limb movement disorder, hypersomnia, parasomnia, and so on). Research adopting ballistocardiography as a way to measure detailed sleep stages began in the 
2010s [9,19-21]. Early studies using ballistocardiographic signals showed a high accuracy of $82 \%$, but most of these studies classified sleep stages briefly as sleep states and wake states or as NREM and REM stages [19]. Tuominen et al. [20] conducted a study using a commercial Beddit sleep tracker to monitor sleep architecture. Consensus in sleep stage classification between the Beddit sleep tracker and polysomnography was very poor when using a four-stage classification (wake, stage N1, N2, and N3 sleep). The authors concluded that it would be currently inapplicable to distinguish between sleep stages. Migliorini et al. [9] classified sleep stages as NREM, REM, and wake state and assessed automatic sleep classification based on heart rate variability, respiration, and movement signals using multi-channel electromechanical film technology (EMFIT) sensors. The agreement between their results and sleep stage classification using polysomnography was kappa $=0.55$. They suggested that their proposed algorithm offers an interesting possibility for sleep monitoring at home. Kortelainen et al. [21] also used EMFIT sensors to evaluate sleep macrostructure using heartbeat intervals and movement activity data and analyzed 18 recordings from healthy subjects using reference polysomnography. When considering the wake, NREM, and REM stages, they obtained a total accuracy of $79 \%$ and kappa $=0.44$. The results of their study indicated that bed sensors may be suitable for monitoring sleep macrostructure in an unobtrusive, low cost, and simple manner. When comparing the proposed algorithm with their algorithm, there is a time-variant autoregressive model for the feature extractor, and a hidden Markov model is used as the classifier. Their algorithm requires an additional movement activity signal and threshold value to classify the wake stage as an input; however, the proposed algorithm does not require an additional movement activity signal and threshold value to identify the wake stage due to the nature of its design.

This study had several limitations. First, although this study focused on detecting sleep stages using vital signs, blood pressure was not studied. There are reports that blood pressure is related to the sleep stages; however, it is not easy to measure in a contactless manner [22]. In fact, contactless methods for blood pressure measurement have been introduced, but these have not been widely commercialized [23]. Further clinical studies on the non-contact measurement of blood pressure and the association between blood pressure and sleep stages are needed. Second, the number of participants in this study was relatively small. In addition, five out of the 35 subjects were excluded from this analysis due to inadequate data. Therefore, in order to determine the reliability of the device and obtain accurate outcomes, it will be essential to increase the number of participants in future studies. Lastly, most participants in this study had OSA, which might have contributed to the moderate agreement seen for sleep architecture. Further studies with healthy controls will provide more reliable results and provide comparative data in patients with OSA.

\section{Conclusion}

The outcomes of this study suggested that there was almost perfect agreement in vital signs such as heart rate, respiratory rate, and body temperature between contactless and contact measurements. In addition, moderate agreement in sleep architecture was observed between the two measurements. These outcomes indicate that the HoneyCube System is a good candidate device for sleep monitoring at home and in multiple environments.

\section{Availability of Data and Material}

The datasets generated or analyzed during the study are available from the corresponding author on reasonable request.

\section{Author Contributions}

Conceptualization: Choi JH, Lee Young Jun, Ha TK, Lee HD. Data curation: Choi JH, Lee Young Jeong, Lee Young Jun. Formal analysis: Moon JE. Funding acquisition: Choi JH Investigation: Lee Young Jeong, HT Kang. Supervision: Choi JH. Writing_original draft: Lee Young Jeong, HT Kang. Writing_-review \& editing: Choi JH.

\section{Conflicts of Interest}

Lee Young Jun, Ha TK, and Lee HD worked for Honeynaps Co., Ltd. The other authors have no financial conflicts of interest relevant to this article.

\section{Funding Statement}

This study was supported by Honeynaps Co., Ltd.

\section{REFERENCES}

1. Mok WQ, Wang W, Liaw SY. Vital signs monitoring to detect patient deterioration: an integrative literature review. Int J Nurs Pract 2015;21 Suppl 2:91-8.

2. Foldvary-Schaefer NR, Waters TE. Sleep-disordered breathing. Continuum (Minneap Minn) 2017;23:1093-116.

3. Lyons MM, Bhatt NY, Pack AI, Magalang UJ. Global burden of sleepdisordered breathing and its implications. Respirology 2020;25:690702 .

4. Ralls F, Cutchen L. A contemporary review of obstructive sleep apnea. Curr Opin Pulm Med 2019;25:578-93.

5. He K, Kapur VK. Sleep-disordered breathing and excessive daytime sleepiness. Sleep Med Clin 2017;12:369-82.

6. Yoshihisa A, Takeishi Y. Sleep disordered breathing and cardiovascular diseases. J Atheroscler Thromb 2019;26:315-27.

7. Vogt E, MacQuarrie D, Neary JP. Using ballistocardiography to measure cardiac performance: a brief review of its history and future significance. Clin Physiol Funct Imaging 2012;32:415-20.

8. Sadek I, Biswas J, Abdulrazak B. Ballistocardiogram signal processing: a review. Health Inf Sci Syst 2019;7:10.

9. Migliorini M, Bianchi AM, Nisticò D, Kortelainen J, Arce-Santana E, Cerutti S, et al. Automatic sleep staging based on ballistocardiographic signals recorded through bed sensors. Annu Int Conf IEEE Eng Med Biol Soc 2010;2010:3273-6.

10. Sidikova M, Martinek R, Kawala-Sterniuk A, Ladrova M, Jaros R, Danys $\mathrm{L}$, et al. Vital sign monitoring in car seats based on electrocardiography, ballistocardiography and seismocardiography: a review. Sensors (Basel) 2020;20:5699.

11. Leonhardt S, Leicht L, Teichmann D. Unobtrusive vital sign monitoring in automotive environments-a review. Sensors (Basel) 2018;18:3080.

12. Berry RB, Quan SF, Abreu AR, Bibbs ML, DelRosso L, Harding SM, et al.; for the American Academy of Sleep Medicine. The AASM manual for the scoring of sleep and associated events: rules, terminology and 
technical specifications, version 2.6. Darien: American Academy of Sleep Medicine 2020.

13. Landis JR, Koch GG. The measurement of observer agreement for categorical data. Biometrics 1977;33:159-74.

14. McGraw KO, Wong SP. Forming inferences about some intraclass correlation coefficients. Psychol Methods 1996;1:30-46.

15. Xie Q, Wang M, Zhao Y, He Z, Li Y, Wang G, et al. A personalized beatto-beat heart rate detection system from ballistocardiogram for smart home applications. IEEE Trans Biomed Circuits Syst 2019;13:1593-602.

16. Albukhari A, Lima F, Mescheder U. Bed-embedded heart and respiration rates detection by longitudinal ballistocardiography and pattern recognition. Sensors (Basel) 2019;19:1451.

17. Sadek I, Heng TTS, Seet E, Abdulrazak B. A new approach for detecting sleep apnea using a contactless bed sensor: comparison study. J Med Internet Res 2020;22:e18297.

18. Wusk G, Gabler H. Non-invasive detection of respiration and heart rate with a vehicle seat sensor. Sensors (Basel) 2018;18:1463.

19. Guerrero-Mora G, Palacios E, Bianchi AM, Kortelainen J, Tenhunen M, Himanen SL, et al. Sleep-wake detection based on respiratory signal acquired through a pressure bed sensor. Annu Int Conf IEEE Eng Med Biol Soc 2012;2012:3452-5.

20. Tuominen J, Peltola K, Saaresranta T, Valli K. Sleep parameter assessment accuracy of a consumer home sleep monitoring ballistocardiograph beddit sleep tracker: a validation study. J Clin Sleep Med 2019; 15:483-7.

21. Kortelainen JM, Mendez MO, Bianchi AM, Matteucci M, Cerutti S. Sleep staging based on signals acquired through bed sensor. IEEE Trans Inf Technol Biomed 2010;14:776-85.

22. Jafari B. Sleep architecture and blood pressure. Sleep Med Clin 2017; 12:161-6.

23. Jeong IC, Finkelstein J. Introducing contactless blood pressure assessment using a high speed video camera. J Med Syst 2016;40:77. 